\title{
Shape and Volumetric Differences in the Corpus Callosum between Patients with Major Depressive Disorder and Healthy Controls
}

\author{
Sekwang Lee ${ }^{1}$, Sung-Bom Pyun ${ }^{2,3}$, Kwan Woo Choi ${ }^{4 凶}$, and Woo-Suk Tae ${ }^{3 凶}$ \\ ${ }^{1}$ Department of Biomedical Sciences, Korea University College of Medicine, Seoul, Republic of Korea \\ ${ }^{2}$ Department of Physical Medicine and Rehabilitation, Korea University College of Medicine, Seoul, Republic of Korea \\ ${ }^{3}$ Brain Convergence Research Center, Korea University Anam Hospital, Seoul, Republic of Korea \\ ${ }^{4}$ Department of Psychiatry, Korea University College of Medicine, Seoul, Republic of Korea
}

Objective This study aimed to investigate the morphometric differences in the corpus callosum between patients with major depressive disorder (MDD) and healthy controls and analyze their relationship to gray matter changes.

Methods Twenty female MDD patients and 21 healthy controls (HCs) were included in the study. To identify the difference in the regional gray matter concentration (GMC), VBM was performed with T1 magnetic resonance imaging. The shape analysis of the corpus callosum was processed. Diffusion tensor imaging (DTI) fiber-tracking was performed to identify the regional tract pathways in the damaged corpus callosal areas.

Results In the shape analysis, regional shape contractions in the rostrum and splenium were found in the MDD patients. VBM analysis showed a significantly lower white matter concentration in the genu and splenium, and a significantly lower GMC in the frontal, limbic, insular, and temporal regions of the MDD patients compared to the HCs. In DTI fiber-tracking, the fibers crossing the damaged areas of the genu, rostrum, and splenium were anatomically connected to the areas of lower GMC in MDD patients.

Conclusion These findings support that major depressive disorder may be due to disturbances in multiple neuronal circuits, especially those associated with the corpus callosum.

Psychiatry Investig 2020;17(9):941-950

Key Words Major depressive disorder, Corpus callosum, Voxel-based morphometry, Magnetic resonance imaging, Shape analysis.

\section{INTRODUCTION}

Major depressive disorder (MDD) is a psychiatric disorder characterized by the dysregulation of mood, cognition, and behavior. ${ }^{1,2}$ Despite the high clinical prevalence and social impact of the disease, its pathogenesis has not yet been elucidated due to the diversity of clinical features and etiologic heterogeneity. ${ }^{3,4}$ To identify the neurobiological mechanism of MDD, several magnetic resonance imaging (MRI) studies

\footnotetext{
Received: April 29, 2020 Revised: June 20, 2020

Accepted: July 29, 2020

$\square$ Correspondence: Woo-Suk Tae, $\mathrm{PhD}$

Brain Convergence Research Center, Korea University Anam Hospital, 73 Inchon-ro, Seongbuk-gu, Seoul 02841, Republic of Korea

Tel: +82-2-2286-1055, Fax: +82-0504-202-4629, E-mail: wstae@korea.ac.kr

$\square$ Correspondence: Kwan Woo Choi, MD

Department of Psychiatry, Korea University College of Medicine, 73 Inchon-ro, Seongbuk-gu, Seoul 02841, Republic of Korea

Tel: +82-2-920-5505, Fax: +82-0504-227-5493

E-mail: choikwanwoo@gmail.com

(c) This is an Open Access article distributed under the terms of the Creative Commons Attribution Non-Commercial License (https://creativecommons.org/licenses/bync/4.0) which permits unrestricted non-commercial use, distribution, and reproduction in any medium, provided the original work is properly cited.
}

have tried to assess the structural brain alterations of patients with MDD. ${ }^{5-9}$ In particular, voxel-based morphometry (VBM) has been applied to investigate subtle alterations in brain regions. VBM is a fully automated technique that is useful in analyzing morphological brain MRI data; it minimizes operational bias and accurately analyzes changes in the concentration or regional volume of brain tissues. ${ }^{10}$ Previous VBM studies in MDD patients reported abnormalities in various regions, including the anterior cingulate cortex (ACC) ${ }^{11-13}$ right middle and inferior frontal gyri, the orbital frontal cortex (OFC), ${ }^{12}$ parahippocampal gyrus, and hippocampus, ${ }^{12}$ as well as widespread gray matter alterations. ${ }^{14-16}$ Other VBM studies revealed subcortical structure changes and suggested that the connection between the cortical and subcortical areas was associated with the dysregulation of emotion processing in MDD patients. ${ }^{17-20}$ However, limited studies have investigated the volumetric or shape alterations of the connection between the cortical and subcortical regions, including the corpus callosum.

The corpus callosum is a major neural pathway connecting 
two cerebral hemispheres and is involved in the integration of motor-sensory and cognitive functions. ${ }^{21}$ This integrative function of the corpus callosum suggests that regional changes in this structure have a significant impact on the integrity of brain function. Recently, several studies have reported that the corpus callosum is one of the important structures related to the pathophysiology of $\mathrm{MDD}^{22-25}$ Despite the alterations in gray matter and corpus callosum reported in MDD patients, the anatomical relationship between these two changes has not been clearly reported. However, in studies analyzing multiple sclerosis patients, anatomical correspondence between white matter and gray matter damage was reported using VBM and tract-based spatial statistics. In addition, a significant correlation between corpus callosum atrophy and gray matter atrophy was also reported. ${ }^{26,27}$ According to one in vitro study, an anatomical connection between the subregion of the corpus callosum and the regional cortical area was revealed. ${ }^{28}$ Other studies reported that regional abnormalities of the cerebral cortex may trigger the atrophy of the corresponding subregions of the corpus callosum. ${ }^{29-31}$ These findings suggest an anatomical link between the area of gray matter change and the location of changes in the corpus callosum in MDD patients.

Surface-based vertex analysis (SVA) allows a quantitative evaluation of brain structures by assessing the relative distances among corresponding surfaces and helps examine the shape changes within the subcortical and cortical areas. ${ }^{32}$ Therefore, SVA analysis can be used to determine which subregions of the brain contribute most to the changes in brain volume and symptoms in psychiatric disorders, such as MDD. ${ }^{33}$ Also, we can effectively analyze the shape changes of specific brain structures, including the corpus callosum using this technique. ${ }^{34}$

In the present study, we hypothesized that there might be significant volumetric and morphometric differences in the corpus callosum between patients with depression and healthy controls (HCs). To test this hypothesis, we investigated the volumetric and morphometric differences between MDD patients and age-matched HCs using optimized VBM and SVA methods. Additionally, we investigated whether changes in corpus callosum and gray matter in MDD had an anatomical relationship to each other. ${ }^{35}$

\section{METHODS}

\section{Participants}

Twenty female patients with MDD were recruited from the department of psychiatry. A board-certified psychiatrist diagnosed MDD based on the Structured Clinical Interview for DSM-IV-TR Axis I Disorders (SCID-I) in the Diagnostic and
Statistical Manual of Mental Disorders, 4th Edition, Text Revision (DSM-IV-TR). ${ }^{36}$ The exclusion criteria were: 1 ) a history of Axis I or Axis II disorders within the last 6 months; 2) psychotic features; 3 ) major neurological illness, such as cerebrovascular disorder or epilepsy; 4) major medical illness, and 5) contraindications to MRI. The duration of the major depressive episodes was evaluated using a life-chart methodology in the interview. Twenty-one age-matched female healthy controls (HCs) were recruited from the community. The HCs received full psychiatric assessment by two psychiatrists for the detection of a history of Axis I or Axis II disorders. The exclusion criteria for the HC group were the same as the patient group. The Korean version of the 17-item Hamilton Depression Rating Scale (HAM-D 17) was performed to evaluate the severity of the depression of the participants on the day of MRI imaging. ${ }^{37,38}$ Self-reported symptoms were also assessed by the Beck Depression Inventory (BDI) ${ }^{39,40}$ All patients were taking antidepressant medications without antipsychotics or mood stabilizers. The study protocol was approved by the Institutional Review Board of the hospital, and all methods in this study were carried out following the approved guidelines and the Declaration of Helsinki. All participants provided written informed consent to participate in the study after receiving a full explanation and understanding of the study.

\section{MRI acquisition}

All the participants were scanned with a 1.5-T Philips Gyroscan MRI scanner (Philips Medical Systems, Best, The Netherlands). Pilot images of the fast field echo T1 MRIs were acquired for consistent head positioning of the subjects with slice thickness $(\mathrm{ST})=10$ millimeters $(\mathrm{mm})$; gap $=10 \mathrm{~mm}$; repetition time $(\mathrm{TR})=15$ milliseconds $(\mathrm{ms})$; echo time $(\mathrm{TE})=5.2$ $\mathrm{ms}$; matrix $=256 \times 256$; and field of view $(\mathrm{FOV})=250 \times 250 \mathrm{~mm}$, with three slices in each orientation and a flip angle (FA) of $20^{\circ}$. Guided by the pilot images, coronal 3D T1-weighted turbo field echo (TFE) MRIs were acquired with the following parameters: $\mathrm{ST}=1.3 \mathrm{~mm}$, no gap, number of slices $=160$ slices, $\mathrm{TR}=10 \mathrm{~ms}, \mathrm{TE}=4.3 \mathrm{~ms}$, number of signal averages $=1$, matrix $=$ $256 \times 256, F O V=220 \times 220 \mathrm{~mm}$, and $\mathrm{FA}=8^{\circ}$. Based on the long axis of the anterior commissure to the posterior commissure from the midsagittal pilot, coronal slices perpendicular to the long axis were obtained.

For fiber tractography, one healthy control was enrolled for diffusion tensor imaging (DTI). MRI was performed using a Siemens MAGNETOM Prisma 3T MRI scanner (Siemens Healthineers, Erlangen, Germany) with a 64-channel head coil with the following parameters for the anatomical images: 3D-MPRAGE sequence with sagittal slices, TR/TE= $2,300 / 2.26 \mathrm{~ms}$, inversion time $(\mathrm{T} 1)=900 \mathrm{~ms}, \mathrm{FA}=8^{\circ}, \mathrm{FOV}=256 \times$ $256 \times 192 \mathrm{~mm}$, slice thickness $=0.5 \mathrm{~mm}$, and voxel size $=$ isotropic 
$0.5 \mathrm{~mm}^{3}$. For the DTI, the parameters were: multiband diffusion-weighted echo-planar imaging with axial slices, TR/TE= $2,137 / 48 \mathrm{~ms}, \mathrm{FA}=90^{\circ}, \mathrm{FOV}=224 \times 224 \times 162 \mathrm{~mm}$, slice thickness $=2.0 \mathrm{~mm}$, voxel size $=1.0 \times 1.0 \times 2.0 \mathrm{~mm}^{3}$, and 128 gradient directions ( $b$-value $=1,000 \mathrm{~s} / \mathrm{mm}^{2}$ ) with the first two and last $31 \mathrm{~b} 0$ images.

\section{Voxel-based morphometry}

Using SPM12 (Wellcome Department of Cognitive Neurology, Institute of Neurology, University College London) implemented in MATLAB (MathWorks, Natick, MA, USA), we performed optimized voxel-based morphometry to investigate the abnormalities in gray/white matter concentrations or regional volume changes. ${ }^{10}$ All MR images of the normal controls and MDD patients were spatially normalized to a standard T1 template to create customized templates and prior images of gray matter, white matter, and cerebrospinal fluid (CSF). Segmentation from the normalized images into gray matter, white matter, and CSF was performed, and the segmented images were sub-sampled into a $2 \mathrm{~mm}^{3}$ voxel cube. The Hidden Markov random field (HMRF) model was used in all segmentation processes to eliminate separated voxels of one tissue class, not likely to be the part of this tissue type. ${ }^{41}$ The spatially normalized images (raw images, segmented gray matter, white matter, and CSF images) were averaged and saved as customized images of the T1 template, gray matter, white matter, or CSF prior images. Lastly, the customized images were smoothed using an isotropic Gaussian kernel with $8 \mathrm{~mm}$ fullwidth at half-maximum (FWHM).

The raw T1 images of all participants were segmented into three partitions (gray matter, white matter, and CSF) automatically in the native space, and the gray and white matter volumes from the images were estimated. The parameters of spatial normalization were estimated by matching individual gray matter with its gray matter template. Spatially normalized images of the original images were generated after the estimation. We segmented the spatially normalized images by the HMRF operation and their prior images (gray, white matter, and CSF partitions). The unmodulated images were smoothed using a $12 \mathrm{~mm}$ full width at half maximum (FWHM) isotropic Gaussian kernel (IGK). Unmodulated VBM is indicative of gray matter or white matter concentration differences. We utilized the Montreal Neurological Institute (MNI) coordinate system to define the coordinates and named the cluster regions according to the atlas of Henri M. Duvernoy. ${ }^{42}$ We used the normalized T1 MRI of a normal control as the template in the figures. We also showed all of the images in the neurological views (the right of the image is the right hemisphere of the brain).

\section{Shape analysis of the corpus callosum}

The T1 MRIs were up-sampled to $0.86 \mathrm{~mm}$ isotropic voxels and located to the $\mathrm{X}, \mathrm{Y}$, and $\mathrm{Z}$ axes at the level of the interhemispheric fissure to acquire exact midsagittal MR images. The reconstructed sagittal images were rotated parallel to the anterior-posterior commissure line. The reconstructed midsagittal MR images were snapped and stored as two-dimensional images. The outline of the corpus callosum was traced manually using ITKSNAP (www.itksnap.org). ${ }^{43}$ For consistent measurements, a tracing line was drawn on the voxels of the mean intensity between the CSF and the corpus callosum.

Shape analysis was processed using the spherical harmonic basis function-point distribution model (SPHARM-PDM), based on a uniform icosahedron-subdivision of the spherical parameterization. ${ }^{33}$ This boundary shape analysis method is useful for the expression of shape deformation. ${ }^{44,45}$ To reduce the file size, cropped images of the corpus callosum from the segmented binary images were saved as new files. These cropped images were re-sampled from the voxel size of 0.86 $\times 0.86 \times 1.30 \mathrm{~mm}$ to $0.5 \mathrm{~mm}$ isotropic voxels. For SPHARM computation, the subdivision level was set to 10 , and the cutoff degree was set to 12 . The correspondence across all surfaces was computed by the alignment of the first order ellipsoids of all the parameterizations. The SPHARM-PDMs of all corpus callosum were computed.

Before the shape analysis, an averaged corpus callosum template was created using the corpus callosum images of all subjects, and the average template was computed by averaging the $3 \mathrm{D}$ coordinates of the corresponding surface points throughout the groups. ${ }^{45}$ All triangulated meshes of the corpus callosum were aligned spatially to the template with a rigid transform. ${ }^{33,44,45}$

\section{DTI fiber-tracking}

We converted the DTI data from DICOM into a NIFTI format by the dcm2nii function in MRIcron (https://www.nitrc. org/projects/mricron), and b-values and b-vector files of all the participants were generated. The eddy currents and head motion artifacts were corrected using the FMRIB Software Library (FSL) ${ }^{46}$ A DTI data set (b-value, b-vector, and raw image files) was reconstructed using a deterministic diffusion fiber-tracking algorithm ${ }^{47}$ in DSI Studio software (https:// dsi-studio.labsolver.org). The parameters for fiber tractography were minimum $\mathrm{FA}=0.15$, maximum angle $=60^{\circ}$, and minimum $/$ maximum length $=50 / 300 \mathrm{~mm}$. The maximum number of fiber-tracking was set to 100,000 streamlines.

To identify the regional tract pathways of the corpus callosum, the damaged areas of the genu, splenium, and rostrum, which corresponded to the VBM and shape analyses results, were manually traced in the mid-sagittal images of the FA map. 


\section{Statistical analyses}

We performed statistical analyses with IBM SPSS Statistics version 24.0 for Windows (IBM Corp., Armonk, NY, USA). Comparisons of age, BDI, and Hamilton Rating Scale for Depression (HDRS) between the controls and patients groups were performed by an independent t-test (two-tailed). In our study, $\mathrm{p}<0.05$ was considered statistically significant. For VBM analyses, we performed an analysis of covariance (ANCOVA) with the confounder of age to analyze the concentration of the unmodulated gray and white matter images. A false discovery rate (FDR) correction at the $\mathrm{p}<0.05$ level was used to correct for multiple comparisons. ${ }^{48}$ The clusters were excepted when their cluster size was fewer than 200 voxels $\left(k_{E}<200\right.$ voxels).

For the statistical analysis of the shape results, the PDM consisted of coordinates of the corresponding 1,002 points (scaled distances from the template with sign). With the scaled distances of 1,002 corresponding points, the differences between the surfaces in the two groups were tested using Hotelling's T-squared test with an age and intracranial volume (ICV) correction. As a result, significance maps of FDR with corrected $\mathrm{p}<0.05$ values for all of the corresponding 1,002 points were created.

\section{RESULTS}

\section{Characteristics of the participants}

Table 1 shows the clinical and demographical characteristics of the participants in the present study. The patients had a mean $( \pm S D)$ age of $42.5( \pm 13.95)$ years and the HCs had a mean age of $42.3( \pm 10.24)$ years. In the MDD patients, the mean age of the first depressive episode was $31.8( \pm 13.10)$ years, the mean number of lifetime depressive episodes was $7.1( \pm 9.12)$, and the mean duration of illness was $9.5( \pm 11.33)$ years. The MDD patients showed significantly higher BDI scores (MDD vs. HCs; $29.6 \pm 13.88$ vs. $4.8 \pm 3.56, \mathrm{p}<0.0001)$ and HAM-D 17 scores (MDD vs. HCs; $21.7 \pm 8.67$ vs. $1.0 \pm 1.23$, $\mathrm{p}<$ $0.0001)$ compared to the HCs.

\section{Voxel-based morphometry \& shape analysis}

There were no statistically significant differences in the ICV between the patients with MDD and the HCs (MDD vs. HCs; $1393.6 \pm 170.84 \mathrm{~cm}^{3}$ vs. $\left.1395.9 \pm 120.48 \mathrm{~cm}^{3}, \mathrm{p}=0.96\right)$. The WMC in the MDD patients was lower in the genu and splenium of the corpus callosum than in the HCs in the VBM analysis (FDR $\mathrm{p}<0.05$ ) (Figure 1A). The GMC in the MDD patients was lower in the frontal lobe (mid-frontal gyri and gyri rectus), limbic lobe (amygdalae, hippocampi, parahippocampal gyri, cingulate gyri, hypothalamic nuclei, and thalami), short and long insular gyri, and the superior/middle temporal gyri (Table 2, Figure 1C) than in the HCs in the VBM analysis. We did not find any significantly higher WMC or GMC in patients with MDD.

In the shape analysis, regional shape contractions were found in the rostrum and upper area of the splenium of the corpus callosum in the MDD patients (FDR p<0.05) (Figure 1B). This finding was partially concordant with the areas of lower WMC in MDD patients in the VBM analysis.

\section{DTI fiber-tracking}

We experimentally analyzed one $\mathrm{HC}$ to track the fibers of the corpus callosum in this study. The fibers passing through the lower WMC area of the genu (Figure 1A) reached bilaterally to the medial and lateral prefrontal cortices (Figure 2A, red), fibers passing through the contracted area of the rostrum (Figure 1B) reached the limbic orbitofrontal cortices and temporal pole corresponding to the limbic cortex (Figure 2B, orange), and the fibers crossing the contracted area of the splenium were connected to the superior/middle temporal gyri and insular gyri (Figure 2C, green). The fibers crossing damaged corpus callosal areas (Figure 1A and B) were anatomically connected to the areas of lower GMC in MDD patients (Figure 1C).

\section{DISCUSSION}

This study presents significant shape contraction in the rostrum and splenium regions of the corpus callosum, as well as

Table 1. Clinical variables of the study participants

\begin{tabular}{lccc}
\hline & MDD $(\mathrm{N}=20)$ & HCs $(\mathrm{N}=21)$ & $\mathrm{p}$-value \\
\hline Age (years, SD) & $42.5 \pm 13.95$ & $42.3 \pm 10.20$ & 0.98 \\
Onset age of depressive episode (years) & $31.8 \pm 13.10$ & - & - \\
Number of depressive episodes & $7.1 \pm 9.12$ & - & - \\
Illness duration (years) & $9.5 \pm 11.33$ & - & - \\
Number of suicide attempts & $0.6 \pm 0.71$ & - & $<0.0001$ \\
Beck Depression Inventory & $29.6 \pm 13.88$ & $4.8 \pm 3.56$ & $<0.0001$ \\
Hamilton Depression Rating Scale & $21.7 \pm 8.67$ & $1.0 \pm 1.23$ & - \\
\hline
\end{tabular}

MDD: major depressive disorder, HCs: healthy controls, SD: standard deviation 

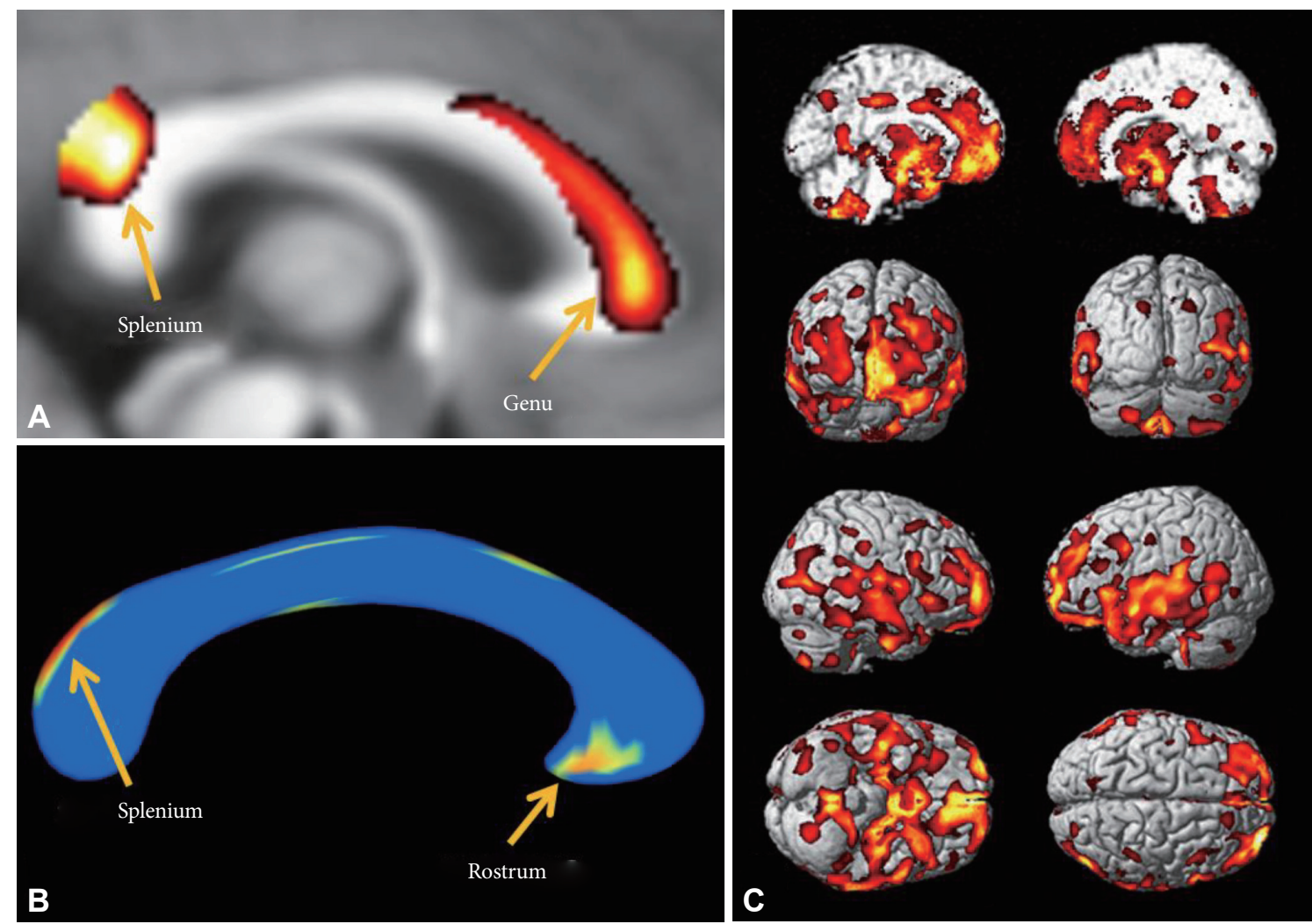

Figure 1. Decreased white matter concentration (WMC) and shape contractions of the corpus callosum in MDD patients. A: In VBM analysis, the WMC in the corpus callosum of MDD patients was decreased in the genu and splenium. B: In the shape analysis, regional shape contractions were found in the rostrum and splenium of the corpus callosum of MDD patients. C: In the VBM analysis, decreased GMC was found in the medial prefrontal, prefrontal, orbitofrontal, cingulate gyri, insular cortices, temporal cortices, and mesial temporal lobes of patients with MDD. The statistical levels were set to a false discovery rate (FDR) correction at the $p<0.05$ level.

significantly lower WMC in the genu and splenium of the corpus callosum of patients with MDD compared to HCs. The present study also showed significantly lower GMC in various brain regions, including the mid-frontal, temporal, cingulate, insular, amygdala, hippocampal, hypothalamic, and thalamic regions in patients with MDD compared to HCs. Additionally, DTI fiber-tracking analysis revealed that the fibers crossing the damaged corpus callosal areas of MDD patients were anatomically associated with the areas of lower GMC in the VBM analysis. Our results revealed altered connectivity between the rostrum, genu, and splenium of the corpus callosum and the associated brain regions, including the frontal lobe, limbic, and other related areas in the MDD patients.

The major finding of this study was the contraction of the regional shape in the rostrum and splenium regions of the corpus callosum in MDD patients compared to HCs. These findings are consistent with previous MRI findings that found smaller corpus callosum volumes in patients with MDD or dysthymia compared to HCs. ${ }^{49-52}$ These studies showed that medication-naïve early-onset dysthymic women ${ }^{49}$ or adolescents and adults with early-onset ( $\leq 18$ years) $\mathrm{MDD}^{51}$ had decreased corpus callosum genu volumes compared to age and sex-matched HCs. When investigating elderly patients (mean age $>60$ years old), Ballmaier et al. ${ }^{52}$ found that callosal thinning was restricted to the genu in the early-onset ( $\leq 60$ years) patients, whereas the patients with late-onset depression showed significant callosal thinning in both the genu and the splenium compared to the HCs. More recently, Dalby et al..$^{53}$ found that subjects with late-onset (after the age of 50 years) MDD displayed significantly higher white matter lesion density in the left superior longitudinal fasciculus and the right frontal projections of the corpus callosum. Consistent with these findings, we found extensive affected lesions in the corpus callosum including the rostrum and splenium, although not in the genu, in middle-aged female MDD patients with recurrent episodes (7.1 \pm 9.12$)$ and relatively long duration of illness $(9.5 \pm$ 11.33 years).

Our study showed significantly lower WMC in the genu and splenium of the corpus callosum in MDD patients via VBM analysis. Consistent with our results, several DTI studies reported lower values of white matter integrity, such as functional anisotropy (FA) values of the corpus callosum, in patients with MDD. ${ }^{24,35,54-60}$ Similar to our results, previous studies showed that middle-aged MDD patients taking med- 
Table 2. Decreased gray matter concentration in patients with major depressive disorder

\begin{tabular}{|c|c|c|c|c|c|c|c|}
\hline \multirow{2}{*}{ Location } & \multicolumn{5}{|c|}{ MNI coordinates (mm) } & \multirow{2}{*}{$\frac{\text { Peak }}{\text { FDR } P^{*}}$} & \multirow{2}{*}{$\begin{array}{c}\text { Peak } \\
\text { Puncorr }\end{array}$} \\
\hline & Side & $\mathrm{x}$ & $\mathrm{y}$ & $\mathrm{z}$ & $\mathrm{T}$ & & \\
\hline Mid-frontal gyrus & $\mathrm{R}$ & 30 & 61 & 18 & 5.20 & 0.004 & $<0.000001$ \\
\hline Mid-frontal gyrus & $\mathrm{L}$ & -31 & 44 & 29 & 4.30 & 0.008 & $<0.00004$ \\
\hline Gyri rectus & $\mathrm{R}$ & 1 & 44 & -29 & 3.53 & 0.019 & 0.001 \\
\hline Gyri rectus & $\mathrm{L}$ & -1 & 11 & -17 & 4.89 & 0.006 & $<0.000001$ \\
\hline Short insular gyrus (anterior) & $\mathrm{R}$ & 49 & -1 & 10 & 5.27 & 0.003 & $<0.000001$ \\
\hline Short insular gyrus (anterior) & $\mathrm{L}$ & -35 & 3 & 9 & 5.58 & 0.003 & $<0.000001$ \\
\hline Long insular gyrus (posterior) & $\mathrm{R}$ & 38 & -18 & 20 & 3.67 & 0.018 & $<0.001$ \\
\hline Long insular gyrus (posterior) & $\mathrm{L}$ & -42 & -33 & 16 & 4.73 & 0.007 & $<0.00001$ \\
\hline Cingulate gyrus (anterior) & B & 3 & 48 & -6 & 4.11 & 0.015 & $<0.001$ \\
\hline Cingulate gyrus (middle) & B & 1 & 5 & 32 & 3.36 & 0.022 & 0.001 \\
\hline Cingulate gyrus (posterior) & $\mathrm{R}$ & 17 & -29 & 43 & 3.86 & 0.015 & $<0.001$ \\
\hline Cingulate gyrus (posterior) & $\mathrm{L}$ & -11 & -28 & 39 & 3.41 & 0.021 & 0.001 \\
\hline Thalami (lateral dorsal neucli) & $\mathrm{B}$ & 2 & -13 & 13 & 2.74 & 0.043 & 0.005 \\
\hline Hypothalamus & $\mathrm{R}$ & 4 & -3 & -9 & 4.04 & 0.013 & $<0.001$ \\
\hline Hypothalamus & $\mathrm{L}$ & -6 & -1 & -8 & 4.68 & 0.007 & $<0.00001$ \\
\hline Amygdala & $\mathrm{R}$ & 25 & -5 & -23 & 4.65 & 0.007 & $<0.00001$ \\
\hline Amygdala & $\mathrm{L}$ & -19 & -6 & -22 & 3.30 & 0.023 & 0.001 \\
\hline Hippocampus & $\mathrm{R}$ & 20 & -11 & -20 & 4.61 & 0.007 & $<0.00001$ \\
\hline Hippocampus & $\mathrm{L}$ & -25 & -11 & -23 & 7.05 & 0.002 & $<0.000001$ \\
\hline Parahippocampal gyrus (anterior) & $\mathrm{R}$ & 39 & -18 & -28 & 4.27 & 0.009 & $<0.00005$ \\
\hline Parahippocampal gyrus (anterior) & $\mathrm{L}$ & -38 & -16 & -32 & 4.70 & 0.007 & $<0.00001$ \\
\hline Lingual gyrus & $\mathrm{L}$ & -32 & -40 & -11 & 3.97 & 0.014 & $<0.001$ \\
\hline Superior/middle/inferior temporal gyrus & $\mathrm{R}$ & 63 & -15 & -14 & 4.07 & 0.014 & $<0.001$ \\
\hline Superior/middle/inferior temporal gyrus & $\mathrm{L}$ & -48 & 2 & -13 & 4.00 & 0.013 & $<0.001$ \\
\hline
\end{tabular}

*false discovery rate (FDR) corrected P, Puncorr: uncorrected P. MNI: montreal neurologic institute, B: bilateral, L: left, R: right, mm: millimeter, extent threshold $\mathrm{kE}>200$ voxels

ication had significantly decreased FA values in the posterior body, ${ }^{57}$ genu, ${ }^{58}$ and splenium ${ }^{54}$ of the corpus callosum compared to HCs. Regarding medication-naïve subjects, similar results of reduced FA values of the genu, ${ }^{56}$ and the genu and the body ${ }^{24}$ of the corpus callosum in middle-aged patients, and decreased FA values of the genu of the corpus callosum in subjects with MDD in their late twenties have been reported. ${ }^{55}$ Recently, studies have been conducted on the role of the splenium in depression, and reductions in the axonal myelin of the splenium in MDD patients was reported. ${ }^{61}$ Also, there have been reports of emotional dysregulation in splenium agenesis. ${ }^{62}$ Regarding the chronicity and recurrent episodes of our participants, chronic and recurrent deteriorations in the whitematter integrity of the corpus callosum might be reflected in WMC reductions in both the genu and splenium of the corpus callosum.

Consistent with numerous previous brain imaging findings, the present study also found significantly lower GMC in various brain regions, including the mid-frontal, ${ }^{5,63}$ temporal, ${ }^{63}$ cingulate, ${ }^{11-13,63}$ insular, ${ }^{63}$ amygdala ${ }^{64-68}$ hippocampal,${ }^{19,69,70}$ hypothalamic, and thalamic ${ }^{71}$ regions in patients with MDD compared to HCs. Our study showed abnormalities of various components (amygdala, cingulate gyrus, and insular cortex) that constitute the limbic system as well as the prefrontal cortex (PFC) of chronic MDD patients. Among them, the amygdala is known as one of the key structures involved in emotion and drive in the limbic system. The cingulate gyrus is above the corpus callosum and constitutes the limbic system. A previous study reported that perigenual anterior cingulate morphology (pACC) was associated with low perceived social status ${ }^{72}$ and sadness and pregenual ACC (pACC). The insular cortex is also known to be involved in the production of emotion. ${ }^{73}$ Our study showed similar results to the anatomical location reported in previous studies, suggesting that several structures are involved in the pathophysiology of MDD. 

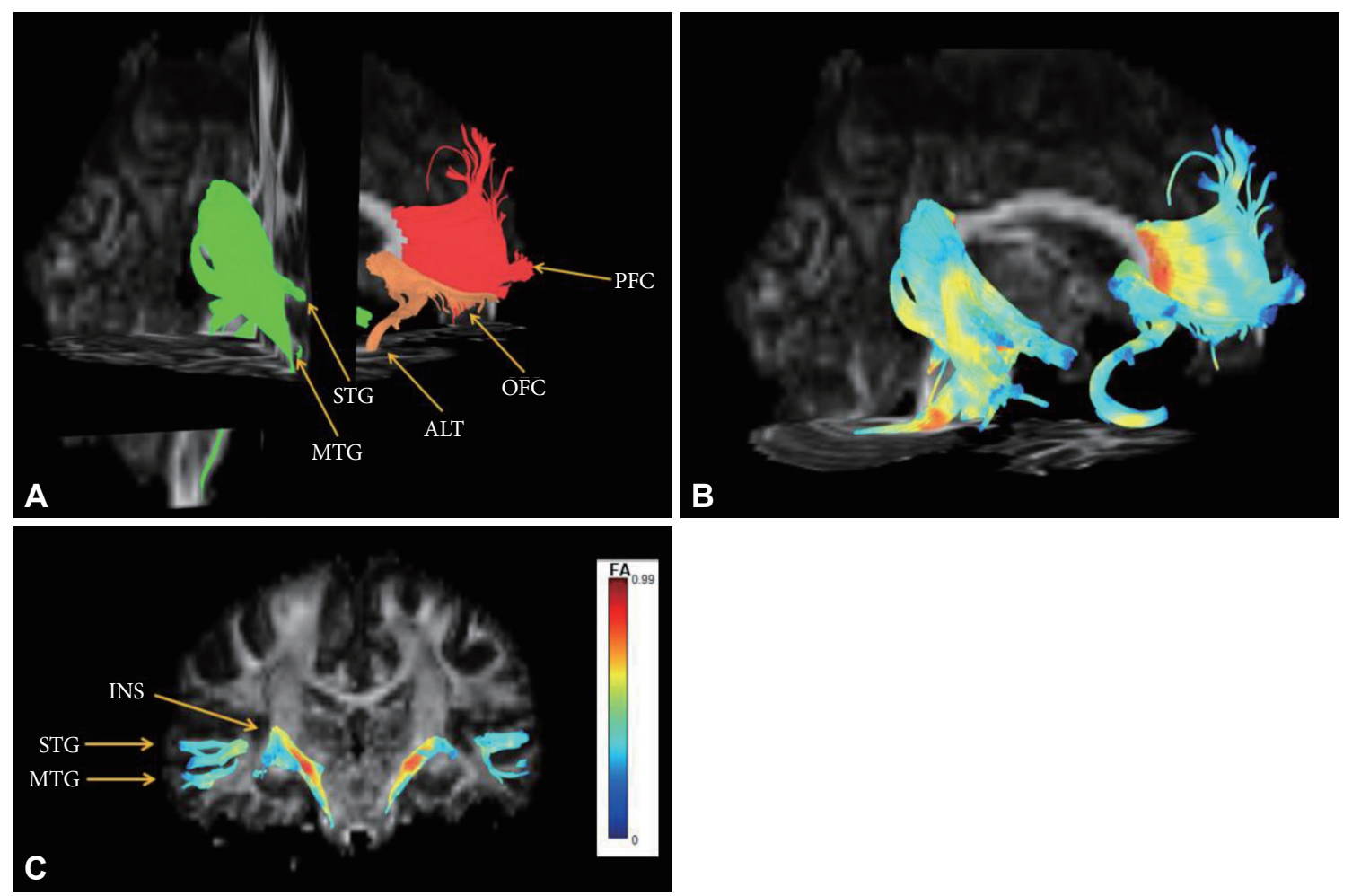

Figure 2. Reconstructed fibers passing through shape-contracted subregions of the corpus callosum. (A) The fibers passing through the decreased WMC area of the genu reached bilaterally to the prefrontal cortices (red), the fibers passing through the contracted area of the rostrum reached the limbic orbitofrontal cortices and temporal pole corresponding to the limbic cortex (orange), and the fibers crossing the contracted area of the splenium connected to the superior/middle temporal gyri and insular gyri (green). (B) The color of the reconstructed fibers represents the fractional anisotropy (FA) values. (C) The color bar represents fractional anisotropy (FA) values.

We also demonstrated a possible connection between corpus callosal areas and the associated cortical areas via DTI analysis of one healthy subject. Using DTI analysis, our results showed that the fibers passing through the genu reached the prefrontal cortex and the fibers passing through the rostrum reached the limbic OFC and temporal pole. Also, this study showed that fibers passing through the splenium reached the temporal and insular gyrus. A possible explanation for this might be that the areas of lower GMC and the areas of lower WMC or regional contraction in the corpus callosum are anatomically correlated, although a causal relationship could not be determined due to the cross-sectional design. According to previous studies, atrophy of the corpus callosum is mainly caused by axonal degeneration due to damage to cortical neurons $^{74,75}$ and the severity of atrophy may be aggravated by cytoskeletal damage in the cerebral white matter. ${ }^{76}$ This mechanism of corpus callosum atrophy supports the presence of an anatomical link between the broad-range gray matter changes and morphologic changes in the corpus callosum in our study. Furthermore, a number of studies have been conducted to examine the changes in neuronal circuits associated with the corpus callosum in patients with depression. A previous positron emission tomography (PET) study revealed an association between depression and decreased activity in the ven- tral prefrontal cortex and the corpus callosum. ${ }^{77}$ Similarly, a meta-analysis of DTI studies performed on MDD patients found that the FA values of the right frontal white matter and the left frontal white matter were decreased in MDD patients and that the main fibers passing through this region were interhemispheric fibers crossing the genu and body of the corpus callosum. ${ }^{60}$ Previous MRI studies found that various cortical regions, such as the prefrontal, orbitofrontal and cingulate areas, and their connections with subcortical areas were associated with emotion and cognitive dysregulation in MDD patients. ${ }^{11,17-20,78}$ Cortico-striatal-pallidal-thalamic (CSPT) brain circuits have been considered to be associated with motor, cognitive, and emotion regulation processes, and related to MDD. ${ }^{79-82}$ Although more studies are needed to explain the association between the subregions of the corpus callosum and its affected areas, this study indicated a possible association between corpus callosal connectivity and cortical areas. Our study suggests that the pathophysiology of MDD is related to alterations in corticolimbic connectivity by showing the significant differences in gray matter concentration across the limbic cortex, hippocampal formation, amygdala, and diencephalon that comprise the limbic system.

The advantage of our study is that we investigated the structural difference between the patients with relatively chronic 
MDD and HCs using a variety of analytical techniques. Although VBM can evaluate both gray and white matter, most VBM studies have mainly analyzed gray matter, and the white matter has been analyzed by other methods known to be more accurate (diffusion tensor imaging, and shape analysis). ${ }^{83}$ The corpus callosum was analyzed by shape analysis and VBM, and the cortical and subcortical differences of the whole brain were analyzed by VBM. Furthermore, a fiber-tracking technique was used to analyze the relationship between the results of the shape analysis and VBM analysis. We could evaluate the MDD pathophysiology not only from the viewpoint of regional brain abnormalities but also from the viewpoint of brain connectivity by comparing the results of each analysis method using a multimodal technique. In addition, we could confirm that the size difference in the subregion was dominant in the rostrum, unlike the genu, which showed a significant difference in white matter concentration, by both techniques. Finally, we tracked the fiber through each subregion of the corpus callosum and confirmed the association between this subregion and the cortical area with reduced GMC.

This study had several limitations and the results should be interpreted cautiously. First, due to the small number of enrolled patients, we could not evaluate differences in the morphometric and volumetric parameters between the subtypes of MDD (e.g., melancholic vs. atypical or familial vs. non-familial $^{22}$ ). To overcome the small sample size, we recruited agematched female subjects. Second, due to the cross-sectional study design, we could not determine the causal relationship between volumetric or morphometric decreases and MDD. Third, all of the patients in our study took antidepressant medications, and this medication itself may have been a confounding factor affecting the results of the study. Fourth, due to the lack of detailed information about the patients' medications, we could not evaluate the effect of medication dosage or the length of pharmacological treatment on the morphological differences in this study. For medication, we evaluated the types of pharmacological treatments that were prescribed for all subjects. However, the treatment duration and dosage were not investigated in this study. Fifth, as all patients were women, these data must be interpreted with caution when generalizing to men. There have been biologically based analyses of why women have a higher incidence and morbidity risk of depressive disorders than men. ${ }^{84,85}$ Further studies are needed to determine whether the neuroanatomical results we observed in women are similar in males. Lastly, since we only evaluated the connectivity between the corpus callosum and the affected cortical areas in one normal control, our results cannot be generalized to the general population. For that reason, future research with more patients is needed to explain the association between the subregions of the corpus callosum and its affected areas.

The present findings revealed regional shape contraction of the corpus callosum and that lower GMC of the PFC and limbic system in patients with MDD compared to HCs may be correlated with the pathophysiology of chronic depression. In particular, lower WMC of the genu and splenium and lower size of the rostrum and splenium in the patients with MDD provide an understanding of depression as a disorder of multiple neuronal circuits, including interhemispheric connectivity of the frontal and temporal lobes.

\section{Acknowledgments}

This work was supported by the National Research Foundation of Korea (NRF) funded by the Korean government (MSIP) (No. 2017R1D1A1B030 30280), the National Research Foundation of Korea (NRF) funded by the Korean government (MSIP) (No. 2017M3C7A1079696), ), a National Research Foundation of Korea (NRF) grant funded by the Korean government (MSIT) (No. 2020R111A1A01067132), and a National Research Foundation of Korea (NRF) grant funded by the Korean government (MSIT) (No. 2020R1C1C1003394). This research was also supported by a grant from the Korea University Anam Hospital, Seoul, Republic of Korea (Grant No. K1922891).

\section{Conflicts of Interest}

The authors have no potential conflicts of interest to disclose.

\section{Author Contributions}

Conceptualization: Woo-Suk Tae. Data curation: Sekwang Lee. Formal analysis: Woo-Suk Tae. Funding acquisition: Kwan Woo Choi and Woo-Suk Tae. Investigation: all authors. Methodology: all authors. Project administration: Woo-Suk Tae and Kwan Woo Choi. Resources: all authors. Software: Sekwang Lee and Woo-Suk Tae. Supervision: Woo-Suk Tae and Sung-Bom Pyun. Validation: Woo-Suk Tae. Visualization: Woo-Suk Tae. Writingoriginal draft: Sekwang Lee. Writing—review \& editing: all authors.

\section{ORCID iDs}

Sekwang Lee

Sung-Bom Pyun

Kwan Woo Choi

Woo-Suk Tae https://orcid.org/0000-0002-3044-2673

https://orcid.org/0000-0002-1933-038X

https://orcid.org/0000-0002-0854-3507 https://orcid.org/0000-0003-0451-0713

\section{REFERENCES}

1. Fava M, Kendler KS. Major depressive disorder. Neuron 2000;28:335341.

2. Hasin DS, Sarvet AL, Meyers JL, Saha TD, Ruan WJ, Stohl M, et al. Epidemiology of adult DSM-5 major depressive disorder and its specifiers in the United States. JAMA Psychiatry 2018;75:336-346.

3. Kessler RC, Berglund P, Demler O, Jin R, Koretz D, Merikangas KR, et al. The epidemiology of major depressive disorder: results from the $\mathrm{Na}$ tional Comorbidity Survey Replication (NCS-R). JAMA 2003;289:30953105 .

4. Baldessarini RJ, Forte A, Selle V, Sim K, Tondo L, Undurraga J, et al. Morbidity in depressive disorders. Psychother Psychosom 2017;86:6572.

5. Drevets WC, Price JL, Furey ML. Brain structural and functional abnormalities in mood disorders: implications for neurocircuitry models of depression. Brain Struct Funct 2008;213:93-118.

6. Marchand WR. Cortico-basal ganglia circuitry: a review of key research and implications for functional connectivity studies of mood and anxiety disorders. Brain Struct Funct 2010;215:73-96. 
7. Marchand WR, Yurgelun-Todd D. Striatal structure and function in mood disorders: a comprehensive review. Bipolar Disord 2010;12:764785 .

8. Mayberg HS. Limbic-cortical dysregulation: a proposed model of depression. J Neuropsychiatry Clin Neurosci 1997;9:471-481.

9. Pizzagalli DA. Frontocingulate dysfunction in depression: toward biomarkers of treatment response. Neuropsychopharmacology 2011;36: 183-206.

10. Ashburner J, Friston KJ. Voxel-based morphometry--the methods. Neuroimage 2000;11(6 Pt 1):805-821.

11. Bora E, Fornito A, Pantelis C, Yucel M. Gray matter abnormalities in Major depressive disorder: a meta-analysis of voxel based morphometry studies. J Affect Disord 2012;138:9-18.

12. Du MY, Wu QZ, Yue Q, Li J, Liao Y, Kuang WH, et al. Voxelwise metaanalysis of gray matter reduction in major depressive disorder. Prog Neuropsychopharmacol Biol Psychiatry 2012;36:11-16.

13. Lai CH. Gray matter volume in major depressive disorder: a meta-analysis of voxel-based morphometry studies. Psychiatry Res 2013;211:3746.

14. Bora E, Fornito A, Pantelis C, Yücel M. Gray matter abnormalities in Major depressive disorder: a meta-analysis of voxel based morphometry studies. J Affect Disord 2012;138:9-18.

15. Grieve SM, Korgaonkar MS, Koslow SH, Gordon E, Williams LM. Widespread reductions in gray matter volume in depression. Neuroimage Clin 2013;3:332-339.

16. Zhao YJ, Du MY, Huang XQ, Lui S, Chen ZQ, Liu J, et al. Brain grey matter abnormalities in medication-free patients with major depressive disorder: a meta-analysis. Psychol Med 2014;44:2927-2937.

17. Koolschijn PC, van Haren NE, Lensvelt-Mulders GJ, Hulshoff Pol HE, Kahn RS. Brain volume abnormalities in major depressive disorder: a meta-analysis of magnetic resonance imaging studies. Hum Brain Mapp 2009;30:3719-3735.

18. Rive MM, van Rooijen G, Veltman DJ, Phillips ML, Schene AH, Ruhe HG. Neural correlates of dysfunctional emotion regulation in major depressive disorder. A systematic review of neuroimaging studies. Neurosci Biobehav Rev 2013;37:2529-2553.

19. Schmaal L, Veltman DJ, van Erp TG, Samann PG, Frodl T, Jahanshad $\mathrm{N}$, et al. Subcortical brain alterations in major depressive disorder: findings from the ENIGMA major depressive disorder working group. Mol Psychiatry 2016;21:806-812.

20. Schmaal L, Hibar DP, Samann PG, Hall GB, Baune BT, Jahanshad N, et al. Cortical abnormalities in adults and adolescents with major depression based on brain scans from 20 cohorts worldwide in the ENIGMA Major Depressive Disorder Working Group. Mol Psychiatry 2017;22:900909.

21. Gazzaniga MS. Cerebral specialization and interhemispheric communication: does the corpus callosum enable the human condition? Brain 2000;123(Pt 7):1293-1326.

22. Lacerda AL, Brambilla P, Sassi RB, Nicoletti MA, Mallinger AG, Frank E, et al. Anatomical MRI study of corpus callosum in unipolar depression. J Psychiatr Res 2005;39:347-354.

23. Walterfang M, Yücel M, Barton S, Reutens DC, Wood AG, Chen J, et al. Corpus callosum size and shape in individuals with current and past depression. J Affect Disord2009;115:411-420.

24. Won E, Choi S, Kang J, Kim A, Han KM, Chang HS, et al. Association between reduced white matter integrity in the corpus callosum and serotonin transporter gene DNA methylation in medication-naive patients with major depressive disorder. Transl Psychiatry 2016;6:e866.

25. Wu JC, Buchsbaum MS, Johnson JC, Hershey TG, Wagner EA, Teng C, et al. Magnetic resonance and positron emission tomography imaging of the corpus callosum: size, shape and metabolic rate in unipolar depression. J Affect Disord 1993;28:15-25.

26. Bodini B, Khaleeli Z, Cercignani M, Miller DH, Thompson AJ, Ciccarelli O. Exploring the relationship between white matter and gray matter damage in early primary progressive multiple sclerosis: an in vivo study with TBSS and VBM. Human Brain Mapp 2009;30:2852-2861.

27. Klawiter EC, Ceccarelli A, Arora A, Jackson J, Bakshi S, Kim G, et al. Corpus callosum atrophy correlates with gray matter atrophy in patients with multiple sclerosis. J Neuroimaging 2015;25:62-67.

28. Witelson SF. Hand and sex differences in the isthmus and genu of the human corpus callosum. A postmortem morphological study. Brain 1989;112(Pt 3):799-835.

29. Moses P, Courchesne E, Stiles J, Trauner D, Egaas B, Edwards E. Regional size reduction in the human corpus callosum following pre- and perinatal brain injury. Cereb Cortex 2000;10:1200-1210.

30. Pelletier J, Suchet L, Witjas T, Habib M, Guttmann CR, Salamon G, et al. A longitudinal study of callosal atrophy and interhemispheric dysfunction in relapsing-remitting multiple sclerosis. Arch Neurol 2001;58: 105-111.

31. Hampel H, Teipel SJ, Alexander GE, Horwitz B, Teichberg D, Schapiro $\mathrm{MB}$, et al. Corpus callosum atrophy is a possible indicator of regionand cell type-specific neuronal degeneration in Alzheimer disease: a magnetic resonance imaging analysis. Arch Neurol 1998;55:193-198.

32. Patenaude B, Smith SM, Kennedy DN, Jenkinson M. A Bayesian model of shape and appearance for subcortical brain segmentation. Neuroimage 2011;56:907-922.

33. Styner M, Gerig G, Lieberman J, Jones D, Weinberger D. Statistical shape analysis of neuroanatomical structures based on medial models. Med Image Anal 2003;7:207-220.

34. Tae WS, Kim SS, Lee KU, Nam EC, Choi JW, Park JI. Hippocampal shape deformation in female patients with unremitting major depressive disorder. AJNR Am J Neuroradiol 2011;32:671-676.

35. Tae WS, Ham BJ, Pyun SB, Kang SH, Kim BJ. Current clinical applications of diffusion-tensor imaging in neurological disorders. J Clin Neurol 2018;14:129-140.

36. American Psychiatric Association. Diagnostic and Statistical Manual of Mental Disorders. 4th Ed. Washington, DC: American Psychiatric Press; 1994.

37. Hamilton M. Development of a rating scale for primary depressive illness. Br J Soc Clin Psychol 1967;6:278-296.

38. Yi JS, Bae SO, Ahn YM, Park DB, Noh KS, Shin HK, et al. Validity and reliability of the Korean version of Hamilton Depression Rating Scale (K-HDRS). J Korean Neuropsychiatr Assoc 2005;44:456-465.

39. Salkind MR. Beck depression inventory in general practice. J R Coll Gen Pract 1969;18:267-271.

40. Beck AT, Ward CH, Mendelson M, Mock J, Erbaugh J. An inventory for measuring depression. Arch Gen Psychiatry 1961;4:561-571.

41. Cuadra MB, Cammoun L, Butz T, Cuisenaire O, Thiran JP. Comparison and validation of tissue modelization and statistical classification methods in T1-weighted MR brain images. IEEE Trans Med Imaging 2005;24:1548-1565.

42. Naidich TP, Duvernoy HM, Delman BN, Sorensen AG, Kollias SS, Haacke EM. Duvernoy's atlas of the human brain stem and cerebellum: highfield MRI, surface anatomy, internal structure, vascularization and 3D sectional anatomy. Vienna, Austria: Springer Science \& Business Media; 2009.

43. Yushkevich PA, Piven J, Hazlett HC, Smith RG, Ho S, Gee JC, et al. User-guided 3D active contour segmentation of anatomical structures: significantly improved efficiency and reliability. Neuroimage 2006;31: 1116-1128.

44. Zhao Z, Taylor WD, Styner M, Steffens DC, Krishnan KR, MacFall JR. Hippocampus shape analysis and late-life depression. PLoS One 2008; 3:e1837.

45. Styner M, Lieberman JA, Pantazis D, Gerig G. Boundary and medial shape analysis of the hippocampus in schizophrenia. Med Image Anal 2004;8:197-203.

46. Jenkinson M, Beckmann CF, Behrens TE, Woolrich MW, Smith SM. FSL. Neuroimage 2012;62:782-790.

47. Yeh FC, Verstynen TD, Wang Y, Fernandez-Miranda JC, Tseng WY. Deterministic diffusion fiber tracking improved by quantitative anisotropy. 
PLoS One 2013;8:e80713.

48. Genovese CR, Lazar NA, Nichols T. Thresholding of statistical maps in functional neuroimaging using the false discovery rate. Neuroimage 2002;15:870-878.

49. Lyoo IK, Kwon JS, Lee SJ, Han MH, Chang CG, Seo CS, et al. Decrease in genu of the corpus callosum in medication-naive, early-onset dysthymia and depressive personality disorder. Biol Psychiatry 2002;52: 1134-1143.

50. MacMaster FP, Langevin LM, Jaworska N, Kemp A, Sembo M. Corpus callosal morphology in youth with bipolar depression. Bipolar Disord 2014;16:889-893.

51. Kemp A, MacMaster FP, Jaworska N, Yang XR, Pradhan S, Mahnke D, et al. Age of onset and corpus callosal morphology in major depression. J Affect Disord 2013;150:703-706.

52. Ballmaier M, Kumar A, Elderkin-Thompson V, Narr KL, Luders E, Thompson PM, et al. Mapping callosal morphology in early- and lateonset elderly depression: an index of distinct changes in cortical connectivity. Neuropsychopharmacology 2008;33:1528-1536.

53. Dalby RB, Chakravarty MM, Ahdidan J, Sorensen L, Frandsen J, Jonsdottir KY, et al. Localization of white-matter lesions and effect of vascular risk factors in late-onset major depression. Psychol Med 2010;40: 1389-1399.

54. Korgaonkar MS, Grieve SM, Koslow SH, Gabrieli JD, Gordon E, Williams LM. Loss of white matter integrity in major depressive disorder: evidence using tract-based spatial statistical analysis of diffusion tensor imaging. Hum Brain Mapp 2011;32:2161-2171.

55. Guo WB, Liu F, Xue ZM, Gao K, Wu RR, Ma CQ, et al. Altered white matter integrity in young adults with first-episode, treatment-naive, and treatment-responsive depression. Neurosci Lett 2012;522:139-144.

56. Xu K, Jiang W, Ren L, Ouyang X, Jiang Y, Wu F, et al. Impaired interhemispheric connectivity in medication-naive patients with major depressive disorder. J Psychiatry Neurosci 2013;38:43-48.

57. Kieseppa T, Eerola M, Mantyla R, Neuvonen T, Poutanen VP, Luoma K, et al. Major depressive disorder and white matter abnormalities: a diffusion tensor imaging study with tract-based spatial statistics. J Affect Disord 2010;120:240-244.

58. Choi S, Han KM, Won E, Yoon BJ, Lee MS, Ham BJ. Association of brain-derived neurotrophic factor DNA methylation and reduced white matter integrity in the anterior corona radiata in major depression. J Affect Disord 2015;172:74-80.

59. Bae JN, MacFall JR, Krishnan KR, Payne ME, Steffens DC, Taylor WD. Dorsolateral prefrontal cortex and anterior cingulate cortex white matter alterations in late-life depression. Biol Psychiatry 2006;60:1356-1363.

60. Liao Y, Huang X, Wu Q, Yang C, Kuang W, Du M, et al. Is depression a disconnection syndrome? Meta-analysis of diffusion tensor imaging studies in patients with MDD. J Psychiatry Neurosci 2013;38:49-56.

61. Williams MR, Sharma P, Macdonald C, Pearce RKB, Hirsch SR, Maier $\mathrm{M}$. Axonal myelin decrease in the splenium in major depressive disorder. Eur Arch Psychiatry Clin Neurosci 2019;269:387-395.

62. Palermo S, Andò A, Salatino A, Sirgiovanni S, De Faveri L, Carassa A, et al. Selective emotional dysregulation in splenium agenesis. A case report of a patient with normal cognitive profile. Front Psychol 2019;10: 631-631.

63. Wise T, Radua J, Via E, Cardoner N, Abe O, Adams TM, et al. Common and distinct patterns of grey-matter volume alteration in major depression and bipolar disorder: evidence from voxel-based meta-analysis. Mol Psychiatry 2017;22:1455-1463.

64. van Eijndhoven P, van Wingen G, van Oijen K, Rijpkema M, Goraj B, Jan Verkes R, et al. Amygdala volume marks the acute state in the early course of depression. Biol Psychiatry 2009;65:812-818.

65. Frodl T, Meisenzahl E, Zetzsche T, Bottlender R, Born C, Groll C, et al. Enlargement of the amygdala in patients with a first episode of major depression. Biol Psychiatry 2002;51:708-714.

66. Hamilton JP, Siemer M, Gotlib IH. Amygdala volume in major depressive disorder: a meta-analysis of magnetic resonance imaging studies. Mol Psychiatry 2008;13:993-1000.

67. Kronenberg G, Tebartz van Elst L, Regen F, Deuschle M, Heuser I, Colla M. Reduced amygdala volume in newly admitted psychiatric in-patients with unipolar major depression. J Psychiatr Res 2009;43:11121117.

68. Saleh K, Carballedo A, Lisiecka D, Fagan AJ, Connolly G, Boyle G, et al. Impact of family history and depression on amygdala volume. Psychiatry Res 2012;203:24-30.

69. Arnone D, McIntosh AM, Ebmeier KP, Munafo MR, Anderson IM. Magnetic resonance imaging studies in unipolar depression: systematic review and meta-regression analyses. Eur Neuropsychopharmacol 2012;22:1-16.

70. McKinnon MC, Yucel K, Nazarov A, MacQueen GM. A meta-analysis examining clinical predictors of hippocampal volume in patients with major depressive disorder. J Psychiatry Neurosci 2009;34:41-54.

71. Choi KW, Han KM, Kim H, Kim A, Kang W, Kang Y, et al. Comparison of shape alterations of the thalamus and caudate nucleus between drug-naive major depressive disorder patients and healthy controls. J Affect Disord 2020;264:279-285.

72. Gianaros PJ, Horenstein JA, Cohen S, Matthews KA, Brown SM, Flory JD, et al. Perigenual anterior cingulate morphology covaries with perceived social standing. Soc Cogn Affect Neurosci 2007;2:161-173.

73. Pandya M, Altinay M, Malone DA, Anand A. Where in the brain is depression? Curr Psychiatry Rep 2012;14:634-642.

74. Pantel J, Schröder J, Jauss M, Essig M, Minakaran R, Schönknecht P, et al. Topography of callosal atrophy reflects distribution of regional cerebral volume reduction in Alzheimer's disease. Psychiatry Res 1999;90: 181-192.

75. Yamauchi H, Fukuyama H, Nagahama Y, Katsumi Y, Dong Y, Hayashi T, et al. Atrophy of the corpus callosum, cortical hypometabolism, and cognitive impairment in corticobasal degeneration. Arch Neurol 1998; 55:609-614.

76. Feany MB, Dickson DW. Widespread cytoskeletal pathology characterizes corticobasal degeneration. Am J Pathol 1995;146:1388-1396.

77. Drevets WC, Price JL, Simpson JR, Todd RD, Reich T, Vannier M, et al. Subgenual prefrontal cortex abnormalities in mood disorders. Nature 1997;386:824-827.

78. Sacher J, Neumann J, Fünfstück T, Soliman A, Villringer A, Schroeter ML. Mapping the depressed brain: a meta-analysis of structural and functional alterations in major depressive disorder. J Affect Disord 2012; 140:142-148.

79. Alexander GE, Crutcher MD, DeLong MR. Basal ganglia-thalamocortical circuits: parallel substrates for motor, oculomotor, "prefrontal" and "limbic" functions. Prog Brain Res 1990;85:119-146.

80. Haber SN, Calzavara R. The cortico-basal ganglia integrative network: the role of the thalamus. Brain Res Bull 2009;78:69-74.

81. Peters SK, Dunlop K, Downar J. Cortico-striatal-thalamic loop circuits of the salience network: a central pathway in psychiatric disease and treatment. Front Syst Neurosci 2016;10:104.

82. Bora E, Harrison BJ, Davey CG, Yucel M, Pantelis C. Meta-analysis of volumetric abnormalities in cortico-striatal-pallidal-thalamic circuits in major depressive disorder. Psychol Med 2012;42:671-681.

83. Whitwell JL. Voxel-based morphometry: an automated technique for assessing structural changes in the brain. J Neurosci 2009;29:9661-9664.

84. Gray AL, Hyde TM, Deep-Soboslay A, Kleinman JE, Sodhi MS. Sex differences in glutamate receptor gene expression in major depression and suicide. Mol Psychiatry 2015;20:1057-1068.

85. Piccinelli M, Wilkinson G. Gender differences in depression: critical review. Br J Psychiatry 2000;177:486-492. 